\title{
Hybrid Perovskite at Full Tilt: Structure and Symmetry Relations of the Incommensurately Modulated Phase of Methylammonium Lead Bromide, $\mathrm{MAPbBr}_{3}$
}

\author{
Dennis Wiedemann,* Joachim Breternitz,* Daniel W. Paley, and Susan Schorr
}

Cite This: J. Phys. Chem. Lett. 2021, 12, 2358-2362

Read Online

ACCESS | Lلll Metrics \& More | 国 Article Recommendations ｜（s Supporting Information

ABSTRACT: As energy-conversion materials, organic-inorganic hybrid perovskites remain a research- and finance-intensive topic. However, even for the arguably most iconic representatives, methylammonium and formamidinium lead halides, the crystal structures of several polymorphs have remained undetermined. Herein, we describe the incommensurately modulated structure of $\mathrm{MAPbBr}_{3}$ in $(3+1) \mathrm{D}$ superspace, as deduced from single-crystal X-ray diffractometry despite systematic twinning. Affirming the published average space group, we determined the superspace group $\operatorname{Imma}(00 \gamma) s 00$ with cell parameters of $a=8.4657(9), b=$ $11.7303(12), c=8.2388(8) \AA$, and $\mathbf{q}=0.2022(8) c^{*}$. Via group-subgroup and mode analyses using irreducible representations, we establish symmetry relationships to the well-known cubic and orthorhombic polymorphs and break down distortions into the average tilt system $a^{-} b^{0} a^{-}$ and modulated contributions to tilt and deformation of the $\mathrm{PbBr}_{6}$ coordination polyhedra. Not only does our model fill a long-standing gap in structural knowledge, but it may also serve as a starting point for elucidating other modulated structures within this substance class.

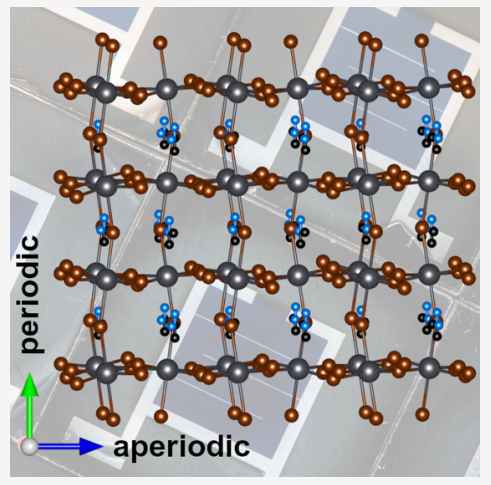

T $\mathrm{n}$ the field of energy-conversion materials, organicinorganic hybrid perovskites are a persistent hot topic that has given rise to extensive research, deduction, prognostication, speculation, and any mix thereof. ${ }^{1,2}$ And while derived perovskite solar cells (PSCs) are already being prototyped and announced for commercialization, many questions concerning underlying crystal-structural principles remain within this surprisingly complex substance class.

Organic-inorganic hybrid perovskites, in the strict sense of the word, ${ }^{3}$ share the formula $A B X_{3}$, with $A$ being a molecular organic cation (typically methylammonium, MA, or formamidinium, FA), $B$ a metal cation (often lead or tin), and $X$ an anion (mostly halide) - although mixed occupation may occur at any position. Most of these compounds come in three perovskite-homeotypic modifications: the cubic high-temperature $\alpha$ phase, the tetragonal medium-temperature $\beta$ phase, and the orthorhombic low-temperature $\gamma$ phase. The crystal structures of these have been characterized with respect to the orientation/position of the organic cation, ${ }^{4}$ their actual spacegroup type, ${ }^{5}$ and their symmetry relationships-and not all facts go undisputed. Besides the rather well-known polymorphs, two further classes of modifications have been described: different $\delta$ phases with non-perovskite structures (e.g., for $\left.\mathrm{FAPbI}_{3}\right)^{6}$ and phases with incommensurately modulated structures, generally dubbed "the incommensurate (IC) phase". The latter occurs at the fringe of the thermally induced $\beta \rightleftarrows \gamma$ transformation, most prominently of $\mathrm{MAPbCl}_{3}$, but has also been reported for methylammonium lead(II) tribromide $\left(\mathrm{MAPbBr}_{3}\right)$ and FA congeners. ${ }^{8,9}$
As early as in 1987 , an intermediate $\mathrm{MAPbBr}_{3}$ phase (then called " $\gamma$ ") at 149.5-155.1 K was reported with a structure conforming to the space group P4/mmm, $a=5.894(2)$ and $c=$ 5.861(2) A, with $Z=1$, as evidenced by indexed GuinierSimon patterns. ${ }^{10}$ Later calorimetric measurements suggested a stability range of $148.8-154.0 \mathrm{~K},{ }^{11}$ before a superstructure in the space-group type $I 4 / \mathrm{mmm}$ with $Z=4$ was suggested based on single-crystal diffractometry. ${ }^{7}$ Incommensurability was postulated in analogy to the chloride congener ${ }^{12}$ but was only thoroughly described by Guo et al. in 2017. ${ }^{13}$ Based on single-crystal X-ray diffraction, they assigned the space-group type Imma $(Z=4)$ to the average structure and estimated a temperature-dependent modulation vector $\mathbf{q}=\gamma \mathbf{c}^{*}$ (referring to the standard orthorhombic setting) with $0.2073 \geq \gamma \geq$ 0.1773 in the range of $148-155 \mathrm{~K}$. Furthermore, the twinning that accompanies the transformations $\beta \rightleftarrows \mathrm{IC} \rightleftarrows \gamma$ was also addressed, but it probably kept the authors from solving the atomic structure of the IC phases. We were able to overcome this problem and present, for the first time, the incommensurately modulated crystal structure of $\mathrm{MAPbBr}_{3}$ at $150 \mathrm{~K}$, where satellite reflections are most pronounced, in the modern $(3+1) \mathrm{D}$ superspace description. ${ }^{4}$

Received: December 18, 2020

Accepted: January 21, 2021

Published: March 5, 2021 
Our investigation is based on a data set previously collected for another study with a different focus. ${ }^{13}$ (The respective crystal had been grown from an aqueous dimethylformamide solution of dimethylamine, hydrogen bromide, and lead(II) iodide using the antisolvent method with 2-propanol as precipitant; see Supplementary Information of ref 13 for details.) Most software packages for data reduction do not support simultaneous handling of twinning and modulation. We were, however, successful in only integrating reflections caused by the major twin domain and dealing with partial overlap during refinement with JANA2006/2020. ${ }^{15}$ The twin law is a rotation of almost $180^{\circ}$ ca. around [101] (i.e., swapping of $\mathbf{a}$ and $\mathbf{c}$, inversion of $\mathbf{b}$ ), which is lost as a symmetry operation during the tetragonal-to-orthorhombic phase transition. The structure conforms to the $(3+1) \mathrm{D}$ superspace group $\operatorname{Imma}(00 \gamma) s 00, a=8.4657(9), b=$ $11.7303(12)$, and $c=8.2388(8) \AA, \mathbf{q}=0.2022(8) \mathrm{c}^{*}$, with $Z$ $=4$, leading to an average structure in the space-group type Imma, as described by Guo et al. before. ${ }^{13}$ Although q equals the rational fraction of $1 /{ }_{5} \mathrm{c}^{*}$ within $3 \sigma$, the authors have shown the modulation vector to vary with temperature. Therefore, we refrain from suggesting a commensurate model but deem a $1 \times 1 \times 5$ cell a suitable approximant (cf. model with starting phase $t_{0}=0$ in the Supporting Information (SI)). The ${ }_{\infty}^{3}\left[\mathrm{PbBr}_{3}\right]^{-}$framework occupies one unique lead and two unique bromide positions (for bond lengths, see Table S2 in the SI). The MA ion was found in two unique perpendicular orientations-one with the $\mathrm{C}-\mathrm{N}$ bond along $\mathbf{b}$, one with it almost along a-in a ratio of $0.33(3): 0.67(3)$. Each of them is disordered over a mirror plane perpendicular to the bond, so that carbon and nitrogen positions are interchangeable. Because only first-order satellite reflections were discernible, modulations (as aforementioned plus displacement modulation for lead and bromide ions) were modeled with harmonic functions of first order. Discontinuous models proved unwarranted, despite slightly larger absolute residual density (cf. Figures S1-S6 in the SI). Compared to routine structure analyses, the quality of the final reduced data $\left(R_{\sigma}=3.52 \%, R_{\text {int }}\right.$ $=12.09 \%)$ and the refined model $\left(R_{1}=8.16 \%, w R_{2}=13.13 \%\right.$, and $S=1.4929$ for all data) suffers from the somewhat approximate treatment of twinning as well as the overall low intensity and overlap of satellite reflections. Keeping these systematic problems in mind, the data and model nonetheless provide a sound basis for the interpretation of the crystal structure and its symmetry relationships (see section 1 in the SI for details on structure solution, refinement, and final model).

The nature of the structural modulation is best understood referring to the average structure (see Figure 1d). Besides minor contributions, the latter is related to the cubic perovskite aristotype structure by two equally large out-of-phase tilts of the $\mathrm{PbBr}_{6}$ coordination octahedra along two $\mathrm{Pb}-\mathrm{Br}-\mathrm{Pb}$ axes (tilt system: $a^{-} b^{0} a^{-}$). This distortion effects a descent from the space group $\operatorname{Pm} \overline{3} m(\mathbf{a}, \mathbf{b}, \mathbf{c})$ to the non-maximal subgroup Imma $(\mathbf{a}+\mathbf{b}, 2 \mathbf{c}, \mathbf{a}-\mathbf{b}$; see Figure 2). Positional modulation is relatively weak for the lead but strong for the bromide ions. Treatment of the MA ion as a rigid molecule showed that the actual position near $0,1,1 / 2$ is governed by hydrogen-bonding to the bromide ions (at least two $\mathrm{NH} \cdots \mathrm{Br}$ bonds per orientation, criteria: $\bar{d}(\mathrm{~N} \cdots \mathrm{Br})<3.8 \AA$ and $\bar{L}(\mathrm{~N}-\mathrm{H} \cdots \mathrm{Br}) \geq$ $140^{\circ}$; see Table S3 in the SI). In this way, the positional modulation of the bromide ions is linked to the translational/
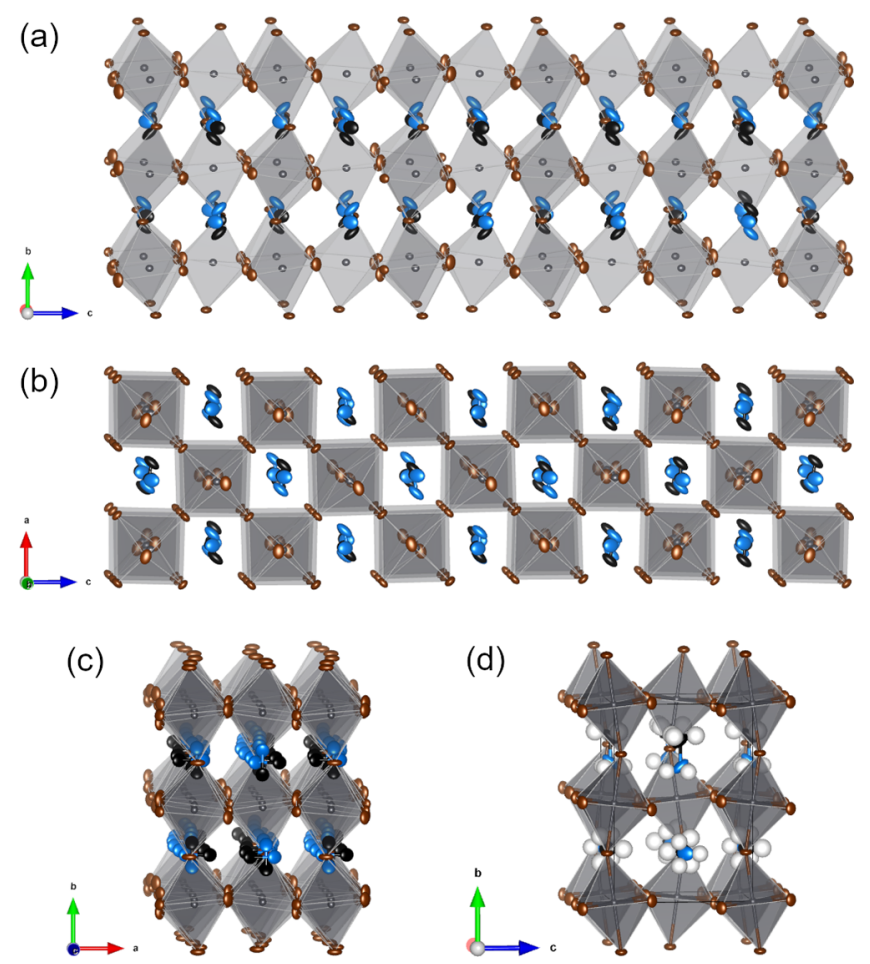

Figure 1. Section from the crystal structure of $\mathrm{IC}-\mathrm{MAPbBr}_{3}$ (approximant with origin at phase $t_{0}=0$, aperiodic along $\mathrm{c}$ ) viewed roughly along (a) $\mathbf{a},(\mathrm{b})-\mathbf{b}$, and (c) $-\mathbf{c}$; hydrogen atoms omitted for clarity, MA ions shown in both unique orientations with arbitrary carbon/nitrogen assignment. (d) Average crystal structure of IC$\mathrm{MAPbBr}$. viewed roughly along a; MA ions only shown in one arbitrary unique orientation for each position with arbitrary carbon/ nitrogen assignment. Atoms are represented as ellipsoids/spheres of $50 \%$ probability (gray, lead; brown, bromine; black, carbon; blue, nitrogen; white, hydrogen) as drawn using VESTA. ${ }^{16}$

rotational modulation of the organic cations. Because of the comparatively low X-ray scattering power and disorder over a position of higher symmetry, we refrain from discussing the MA positions/orientation in more depth and will thus focus on the ${ }_{\infty}^{3}\left[\mathrm{PbBr}_{3}\right]^{-}$framework. The incommensurate modulation of bromide positions mainly affects the tilts of distorted $\mathrm{PbBr}_{6}$ octahedra: While, on average, they conform to $a^{-}$in two directions (see above), they vary harmonically between no tilt and a maximal out-of-phase tilt, phase-shifted by half a period with respect to each other (see Figure 3 and $t$ movie in the SI). A slight twist of the basal $\mathrm{Pb}(\mathrm{Brl})_{4}$ plane, which is tilted with respect to the ac plane, occurs concomitantly. One modulation period spans $1 / \gamma$ cells in the $c$ direction, so that $2 \gamma$ changes per cell between zero and maximum tilt ensue. As $\gamma$ increases with decreasing temperature, ${ }^{13}$ so does the number of changes in a given crystal segment. For $\gamma \rightarrow 1$ or $\gamma \rightarrow 0$, the $a^{-} b^{+} a^{-}$structure of low-temperature $\mathrm{MAPbBr}_{3}$ or a hypothetical $a^{-} b^{0} c^{-}$ structure in $\mathrm{C} 2 / \mathrm{m}(-\mathbf{a}-\mathbf{c},-\mathbf{b}, \mathbf{c})$ would be approached, respectively.

The bromide displacement parameters at each modulation phase reflect the bonding, tilt, and twist situation in a physically sensible manner. While the displacement modulation of $\mathrm{Pb} 1$ is very small but significant, $\mathrm{Br} 1$ and $\mathrm{Br} 2$ exhibit only one significant and large modulation parameter each: the ones of $U_{22}(\mathrm{Br} 1)$ and $U_{13}(\mathrm{Br} 2)$ (cf. $t$ movie in the SI). This means that, for $\mathrm{Br} 1$, the magnitude of displacement along $\mathbf{b}$ increases with bending the (ideally linear) $\mathrm{Pb}-\mathrm{Br}-\mathrm{Pb}$ 


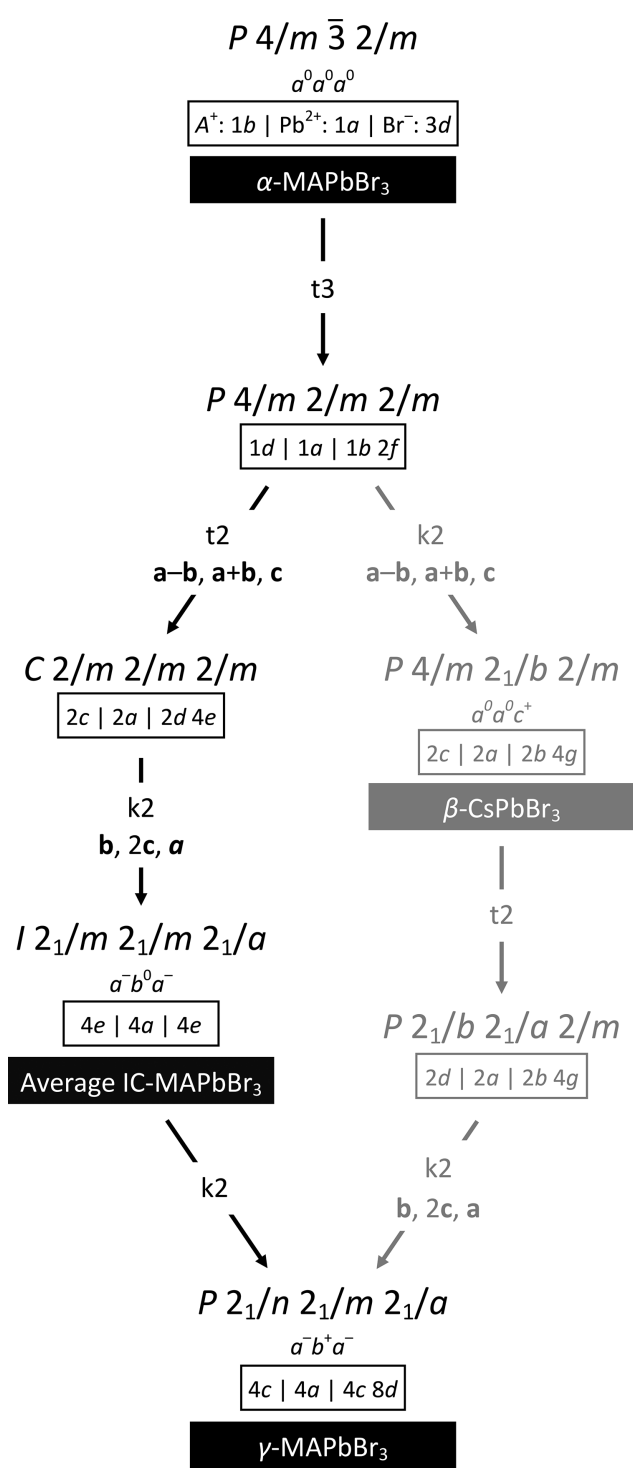

Figure 2. Bärnighausen tree showing the group-subgroup relationships between the discussed structures (discussed path in black, alternative in gray). Nodes consist of full Hermann-Mauguin symbols for the space-group type, tilt systems in Glazer notation (if a representative exists), ${ }^{17}$ occupied Wyckoff positions (by $A^{+}, \mathrm{Pb}^{2+}$ and $\mathrm{Br}^{-}$ions), and a phase representative (if existent, with dark background). Links are annotated with kind and index of relationship as well as transformation of the cell basis (if applicable).

arrangement along $\mathbf{b}$, signifying enlarged anisotropic vibrational motion. For $\mathrm{Br} 2$, on the other hand, the modulation of displacement orientation in the ac plane conveys a dynamic shift toward the nearest hydrogen-bonding donor at each phase.

Analysis of the structural distortion modes induced by irreducible representations of space-group symmetries using ISODISTORT gives further insight into the phase relationships $\alpha \rightarrow \mathrm{IC} \rightarrow \gamma$, especially with respect to $\mathrm{PbBr}_{6}$ tilts and distortions. ${ }^{18,19}$ The main idea of this method is to consider the structural degrees of freedom not in terms of individual atomic coordinates but of collective displacements (the socalled displacive distortion modes) that transform according to the irreducible representations of the higher-symmetry parent space group. (We will not consider strain modes, which convey the rather trivial changes of unit-cell lengths.) For this purpose,

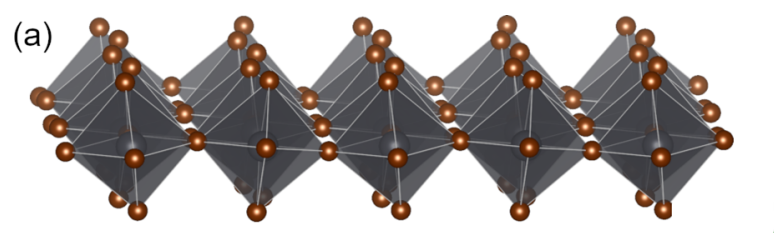

(b)
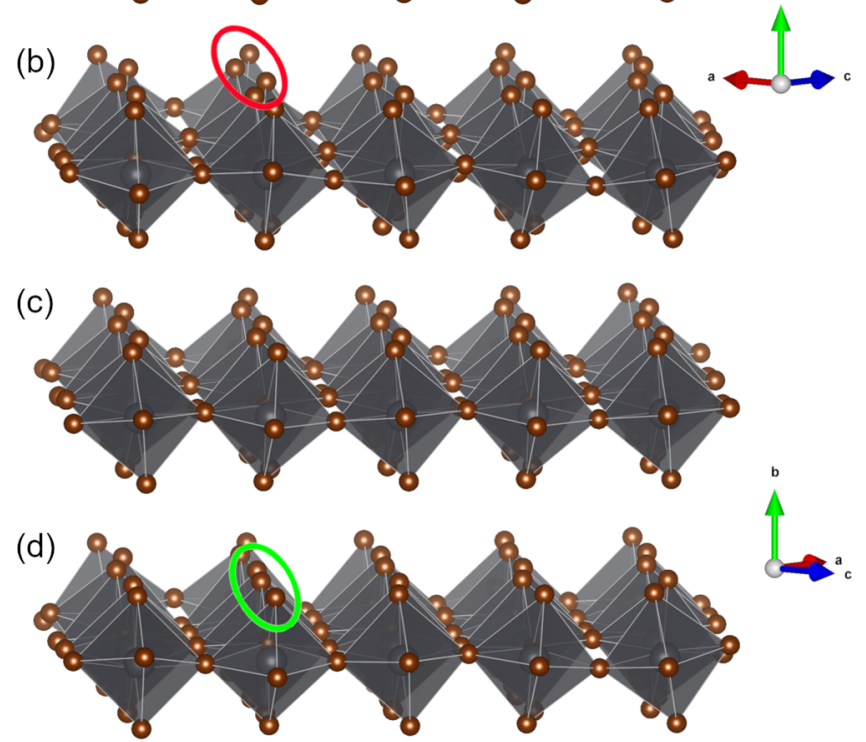

Figure 3. Sections of the average (a, c) and modulated crystal structure at $t_{0}=0(\mathrm{~b}, \mathrm{~d})$ viewed roughly along $\mathbf{a}+\mathbf{c}(\mathrm{a}, \mathrm{b})$ and $\mathbf{a}-\mathbf{c}$ (c, d) corresponding to two main axes of the cubic perovskite aristotype. Atoms are drawn with arbitrary radii (gray, lead; brown, bromine) using VESTA; ${ }^{16}$ MA ions were omitted for clarity. The average structure exhibits the same $a^{-}$out-of-phase tilt in both directions, while the modulated structure varies from almost zero (green circle) to nearly maximal out-of-phase tilt (red circle). Please note that the modulation direction is none of the bisectors but $\mathrm{cllc}^{*}$ (cf. Figure $1 \mathrm{a}-\mathrm{c}$ ).

we have separated the hypothetical transformation of the cubic $\alpha-\mathrm{MAPbBr}_{3}$ aristotype into the average IC- $\mathrm{MAPbBr}_{3}$ structure from the incommensurate distortion into the actual IC$\mathrm{MAPbBr}_{3}$ hettotype (see Figure 4 and the detailed breakdown in Table S4 in the SI). The positional parameters from the structural models were then decomposed into distortion modes and assigned to specific changes in the $\mathrm{PbBr}_{6}$ polyhedron after inspection. Because of its disorder, the MA ion was excluded by placing an invariant pseudoatom in an idealized position. For each transformation, one single major distortion mode was found (transforming like $\mathrm{R}_{4}{ }^{+}, \Lambda_{4}$, and $\mathrm{X}_{4}^{+}$, respectively) and identified as "primary", which means it can effect the symmetry lowering on its own. For $\alpha \rightarrow$ IC (average), this conveys the octahedral $a^{-} b^{0} a^{-}$tilt as described above. Within the orthorhombic system, the $\mathrm{PbBr}_{6}$ environment does not have a strictly octahedral shape and is subject to distortion with respect to an equatorial base (ca. in the ac plane) and an apical axis (ca. along b). A shear of the base and a tilt of the axis, each in two directions, lead to the well-known $\gamma-\mathrm{MAPbBr}_{3}$ in the maximal subgroup type Pnma (thus, this transformation may be of second order according to Landau theory). In the gedankenexperiment of following IC (average) $\rightarrow$ IC, the same incommensurate distortion manifests as tilt or twist / shear or shift, depending on the position in the crystal. Overall, the modulation causes an additional tilt of the $\mathrm{PbBr}_{6}$ polyhedron with concomitant deformation of the base. The reason for the occurrence of incommensurability within a narrow temper- 


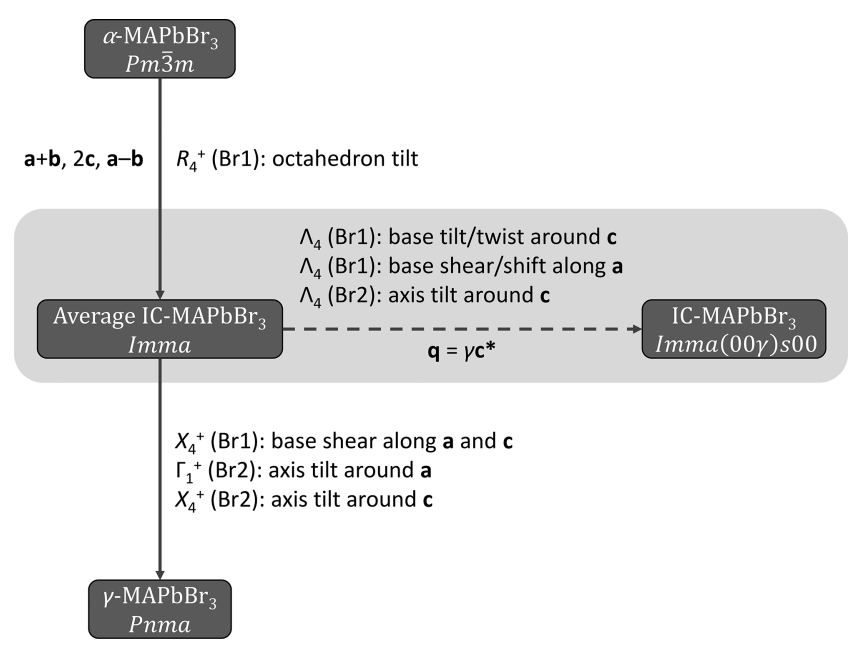

Figure 4. Distortion modes describing the (hypothetical) transformations between different polymorphs of $\mathrm{MAPbBr}_{3}$. Nodes name the polymorph and its (super)space-group type; links are annotated with coordinate transformations as well as irreducible representations, affected atoms, and description of the predominant displacive distortions. "Base" refers to the $\mathrm{Pb}(\mathrm{Br} 1)_{4}$ coordination plane, "axis" to the $\mathrm{Br} 2-\mathrm{Pb}-\mathrm{Br} 2$ arrangement.

ature range seems to be structural frustration during the reconstructive first-order transition $\beta \rightarrow \mathrm{IC}$, which crosses over from another branch of the Bärnighausen tree. This is evidenced by some locally rather unfavorable deformations (globally thinned out via modulation) that relax into the periodic pattern of $\gamma-\mathrm{MAPbBr}_{3}$ upon further cooling.

For our study, we have solved and refined the hitherto unknown incommensurately modulated structure of $\mathrm{MAPbBr}_{3}$ at $150 \mathrm{~K}$ and presented a model of ample quality despite systematic problems. (Crystals suffer from twinning caused by the symmetry descent from $P 4 / \mathrm{mmm}(\mathbf{a}, \mathbf{b}, \mathbf{c})$ to $\mathrm{Cmmm}$ $(\mathbf{a}-\mathbf{b}, \mathbf{a}+\mathbf{b}, \mathbf{c})$ underlying the reconstructive $\beta \rightarrow$ IC transformation.) The average orthorhombic unit cell in the spacegroup type Imma gives rise to a known perovskite variant derived by an $a^{-} b^{0} a^{-}$tilt from the cubic aristotype. ${ }^{20}$ With $a \approx$ $8, b \approx 12$, and $c \approx 8 \AA$, and $Z=4$, the parameters are typical for the system. The actual structure in the $(3+1) \mathrm{D}$ superspace group $\operatorname{Imma}(00 \gamma) s 00, \mathbf{q} \approx 0.2 \mathbf{c}^{*}$, is subject to additional distortions: Via mode analysis, these were identified to transform like the irreducible representation $\Lambda_{4}$ and manifest in an additional incommensurate tilt of the $\mathrm{PbBr}_{6}$ polyhedra and deformations of their equatorial plane. We tentatively assign this to structural frustration induced during the firstorder $\beta \rightarrow \mathrm{IC}$ transformation, which is resolved through restoration of the periodicity in the IC $\rightarrow \gamma$ transformation upon further cooling. Not only do our model and the in-depth analysis of its symmetry relationships shed light on one of the most iconic organic-inorganic hybrid perovskites, but they may also serve as a starting point for pinning down and elucidating other (suspected) modulated structures within this substance class (e.g., of $\mathrm{MAPbCl}_{3},{ }^{7,21} \mathrm{MAPbI}_{3}{ }^{22} \mathrm{FAPbBr}_{3},{ }^{8}$ or $\mathrm{FAPbI}_{3}$ ).

\section{ASSOCIATED CONTENT}

\section{(s) Supporting Information}

The Supporting Information is available free of charge at https://pubs.acs.org/doi/10.1021/acs.jpclett.0c03722.
Movie showing the 3D crystal structure as a function of the cell modulation phase $t$, hydrogen atoms with arbitrary radius, all other atoms as ellipsoids of 50\% probability (gray: $\mathrm{Pb}$, brown: $\mathrm{Br}$, black: $\mathrm{C}$, blue: $\mathrm{N}$; white: $\mathrm{H} ; \mathrm{Br} 1$ and $\mathrm{Br} 2$ in ac plane at $y \approx 0$ and $1 / 2$ or at $y$ $\approx 1$ and $3 / 4$, respectively), MA ions in both unique orientations with arbitrary $\mathrm{C} / \mathrm{N}$ assignment (omitted at ca. $1 / 2,1,1$ and $1 / 2,3 / 4,1$ for clarity), cell borders in gray (MP4)

Experimental and geometrical details, de-Wolff sections, and details on symmetry relationships (PDF)

Basic crystallographic information file for a $2 \times 1 \times 5$ approximant cell with the starting phase $t_{0}=0$, caveat: aperiodic beyond boundaries (CIF)

\section{AUTHOR INFORMATION}

\section{Corresponding Authors}

Dennis Wiedemann - Department Structure and Dynamics of Energy Materials, Helmholtz-Zentrum Berlin für Materialien und Energie GmbH, 14109 Berlin, Germany; 이이.org/ 0000-0001-6294-3205; Email: dennis.wiedemann@

helmholtz-berlin.de

Joachim Breternitz - Department Structure and Dynamics of Energy Materials, Helmholtz-Zentrum Berlin für Materialien und Energie GmbH, 14109 Berlin, Germany; 이이.org/ 0000-0002-0192-6919; Email: joachim.breternitz@ helmholtz-berlin.de

\section{Authors}

Daniel W. Paley - Columbia Nano Initiative, Columbia University, New York 10027, United States

Susan Schorr - Department Structure and Dynamics of Energy Materials, Helmholtz-Zentrum Berlin für Materialien und Energie GmbH, 14109 Berlin, Germany; Institute of Geological Sciences, Freie Universität Berlin, 12249 Berlin, Germany

Complete contact information is available at:

https://pubs.acs.org/10.1021/acs.jpclett.0c03722

\section{Notes}

The authors declare no competing financial interest.

\section{ACKNOWLEDGMENTS}

We acknowledge Dr. Trevor D. Hull and Professor Jonathan S. Owen (Department of Chemistry, Columbia University, United States) for providing single crystals of methylammonium lead bromide. X-ray diffraction was performed in the Shared Materials Characterization Laboratory at Columbia University. We thank Dr. Václav Petříček (Fyzikální ústav AV ČR, Prague, Czech Republic) for providing us with a development version of the program JANA2020 and continuous support.

\section{REFERENCES}

(1) Correa-Baena, J.-P.; Abate, A.; Saliba, M.; Tress, W.; Jesper Jacobsson, T.; Grätzel, M.; Hagfeldt, A. The Rapid Evolution of Highly Efficient Perovskite Solar Cells. Energy Environ. Sci. 2017, 10, 710-727.

(2) Olaleru, S. A.; Kirui, J. K.; Wamwangi, D.; Roro, K. T.; Mwakikunga, B. Perovskite Solar Cells: The New Epoch in Photovoltaics. Sol. Energy 2020, 196, 295-309.

(3) Breternitz, J.; Schorr, S. What Defines a Perovskite? Adv. Energy Mater. 2018, 8, 1802366. 
(4) Franz, A.; Többens, D. M.; Lehmann, F.; Kargell, M.; Schorr, S. The Influence of Deuteration on the Crystal Structure of Hybrid Halide Perovskites: A Temperature-Dependent Neutron Diffraction Study of $\mathrm{FAPbBr}_{3}$. Acta Crystallogr., Sect. B: Struct. Sci., Cryst. Eng. Mater. 2020, 76, 267-274.

(5) Breternitz, J.; Lehmann, F.; Barnett, S. A.; Nowell, H.; Schorr, S. Role of the Iodide-Methylammonium Interaction in the Ferroelectricity of $\mathrm{CH}_{3} \mathrm{NH}_{3} \mathrm{PbI}_{3}$. Angew. Chem., Int. Ed. 2020, 59, 424-428.

(6) Stoumpos, C. C.; Malliakas, C. D.; Kanatzidis, M. G. Semiconducting Tin and Lead Iodide Perovskites with Organic Cations: Phase Transitions, High Mobilities, and Near-Infrared Photoluminescent Properties. Inorg. Chem. 2013, 52, 9019-9038.

(7) Kawamura, Y.; Mashiyama, H. Modulated Structure in Phase II of $\mathrm{CH}_{3} \mathrm{NH}_{3} \mathrm{PbCl}_{3}$. J. Korean Phys. Soc. 1999, 35, 1437-1440. https:// www.jkps.or.kr/journal/view.html?uid=3952 (accessed 01/22/2021).

(8) Elbaz, G. A. Transport Phenomena in Lead Halide Perovskites and Layered Materials. Ph.D. Thesis, Columbia University, New York, 2017. DOI: $10.7916 /$ D80Z7FRK.

(9) Kawachi, S.; Atsumi, M.; Saito, N.; Ohashi, N.; Murakami, Y.; Yamaura, J.-i. Structural and Thermal Properties in Formamidinium and Cs-Mixed Lead Halides. J. Phys. Chem. Lett. 2019, 10, 69676972.

(10) Poglitsch, A.; Weber, D. Dynamic Disorder in Methylammoniumtrihalogenoplumbates (II) Observed by Millimeter-Wave Spectroscopy. J. Chem. Phys. 1987, 87, 6373-6378.

(11) Onoda-Yamamuro, N.; Matsuo, T.; Suga, H. Calorimetric and IR Spectroscopic Studies of Phase Transitions in Methylammonium Trihalogenoplumbates (II). J. Phys. Chem. Solids 1990, 51, 13831395.

(12) Swainson, I. P.; Stock, C.; Parker, S. F.; Van Eijck, L.; Russina, M.; Taylor, J. W. From Soft Harmonic Phonons to Fast Relaxational Dynamics in $\mathrm{CH}_{3} \mathrm{NH}_{3} \mathrm{PbBr}_{3}$. Phys. Rev. B: Condens. Matter Mater. Phys. 2015, 92, 100303.

(13) Guo, Y.; Yaffe, O.; Paley, D. W.; Beecher, A. N.; Hull, T. D.; Szpak, G.; Owen, J. S.; Brus, L. E.; Pimenta, M. A. Interplay between Organic Cations and Inorganic Framework and Incommensurability in Hybrid Lead-Halide Perovskite $\mathrm{CH}_{3} \mathrm{NH}_{3} \mathrm{PbBr}_{3}$. Phys. Rev. Mater. 2017, 1, 042401.

(14) Janssen, T.; Janner, A.; Looijenga-Vos, A.; de Wolff, P. M., Incommensurate and Commensurate Modulated Structures. In International Tables for Crystallography; Prince, E., Ed.; John Wiley \& Sons: Hoboken, NJ, 2006; Vol. C, pp 907-955. DOI: 10.1107/ 97809553602060000624.

(15) Petříček, V.; Dušek, M.; Palatinus, L. Crystallographic Computing System JANA2006: General Features. Z. Kristallogr. Cryst. Mater. 2014, 229, 345-352.

(16) Momma, K.; Izumi, F. VESTA 3 for Three-Dimensional Visualization of Crystal, Volumetric and Morphology Data. J. Appl. Crystallogr. 2011, 44, 1272-1276.

(17) Glazer, A. M. The Classification of Tilted Octahedra in Perovskites. Acta Crystallogr., Sect. B: Struct. Crystallogr. Cryst. Chem. 1972, 28, 3384-3392.

(18) Stokes, H. T.; Hatch, D. M.; Campbell, B. J. ISODISTORT, Isotropy Software Suite; Brigham Young University: Provo, UT, 2020; https://iso.byu.edu.

(19) Campbell, B. J.; Stokes, H. T.; Tanner, D. E.; Hatch, D. M. ISODISPLACE: A Web-Based Tool for Exploring Structural Distortions. J. Appl. Crystallogr. 2006, 39, 607-614.

(20) Howard, C. J.; Stokes, H. T. Group-Theoretical Analysis of Octahedral Tilting in Perovskites. Acta Crystallogr., Sect. B: Struct. Sci. 1998, 54, 782-789.

(21) Chi, L.; Swainson, I.; Cranswick, L.; Her, J.-H.; Stephens, P.; Knop, O. The Ordered Phase of Methylammonium Lead Chloride $\mathrm{CH}_{3} \mathrm{ND}_{3} \mathrm{PbCl}_{3}$. J. Solid State Chem. 2005, 178, 1376-1385.

(22) Jacques, V. L. R.; Gallo-Frantz, A.; Tejeda, A.; Le Bolloc'h, D.; Lédée, F.; Trippé-Allard, G.; Garrot, D.; Fertey, P.; Deleporte, E.; Plantevin, O. A New Long-Range Sub-Structure Found in the Tetragonal Phase of $\mathrm{CH}_{3} \mathrm{NH}_{3} \mathrm{PbI}_{3}$ Single Crystals. J. Phys. D: Appl. Phys. 2019, 52, 314001. 